\title{
Mass sensor for in situ monitoring of focused ion and electron beam induced processes
}

\author{
Vinzenz Friedlia) \\ EMPA, Swiss Federal Laboratories for Materials Testing and Research, Materials and Nanomechanics \\ Laboratory, Feuerwerkerstr. 39, CH-3602 Thun, Switzerland and Ecole Polytechnique Fédérale de Lausanne \\ (EPFL), Institute of Imaging and Applied Optics, CH-1015 Lausanne, Switzerland \\ Christian Santschi \\ Centre Suisse d'Electronique et de Microtechnique (CSEM), Jaquet Droz 1, CH 2002 Neuchâtel, \\ Switzerland

\begin{abstract}
Johann Michler
EMPA, Swiss Federal Laboratories for Materials Testing and Research, Materials and Nanomechanics
\end{abstract} \\ Laboratory, Feuerwerkerstr. 39, CH-3602 Thun, Switzerland \\ Patrik Hoffmann \\ Ecole Polytechnique Fédérale de Lausanne (EPFL), Institute of Imaging and Applied Optics, CH-1015 \\ Lausanne, Switzerland \\ Ivo Utke \\ EMPA, Swiss Federal Laboratories for Materials Testing and Research, Materials and Nanomechanics \\ Laboratory, Feuerwerkerstr. 39, CH-3602 Thun, Switzerland
}

(Received 27 October 2006; accepted 20 December 2006; published online 31 January 2007)

\begin{abstract}
A cantilever-based mass sensor for in situ monitoring of deposition and milling using focused ion and electron beams is presented. Carefully designed experiments allowed for mass measurements with a noise level of $\pm 10 \mathrm{fg}$ by tracking the resonance frequency of a temperature stabilized piezoresistive cantilever using phase locking. The authors report on measurements of precursor surface coverage, residence time, mass deposition rates, yields, and deposit density using the $\left(\mathrm{CH}_{3}\right)_{3} \mathrm{PtCpCH}_{3}$ precursor. () 2007 American Institute of Physics. [DOI: 10.1063/1.2435611]
\end{abstract}

Focused ion and electron beam (FIB and FEB) induced processing (deposition and etching) uses additive or subtractive mechanisms occurring when charged particles impinge on a substrate upon which molecules are adsorbed. Optimization of FIB/FEB induced processes for reliable fabrication and applications in micro- and nanodevices requires further understanding of the basic physics underlying the process. One way to efficient advancement is in situ process monitoring.

Presently, FIB/FEB process monitoring is restricted to shape evolution of deposits and etch progress of holes (vias) based on the analysis of stage current and/or the secondary electron signal. In FIB milling this approach is conventionally used for end point detection. ${ }^{1}$ In FEB induced deposition it is used for continuous shape monitoring and process reproducibility. ${ }^{2}$ Nowadays, in dual beam machines continuous visualization of FIB processing is achieved with live FEB imaging. The method was applied to control gap reduction down to $5 \mathrm{~nm}$ between Pt containing electrodes. ${ }^{3}$ In situ real-time laser reflectometry was employed to measure the evolution of optical thickness of transparent materials deposited by FEB. ${ }^{4}$ With respect to mass evolution quartz crystal microbalances (QCMs) with subnanogram resolution were used for in situ measurement of ion beam induced process yields including the corresponding adsorbed precursor surface coverage. ${ }^{5}$ The work described here is motivated by the insufficient mass resolution of QCMs to measure nanostructures.

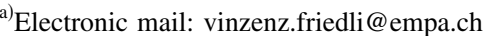

In this letter we show in situ monitoring of precursor adsorption, FIB milling, and FIB/FEB induced deposition rates and yields using cantilever-based mass sensors.

A schematic diagram of the mass sensor is shown in Fig. 1. A silicon cantilever $\left(508 \times 175 \times 8 \mu \mathrm{m}^{3}, f_{\text {res }}=43.4 \mathrm{kHz}\right)$ with an integrated piezoresistive Wheatstone bridge for deflection readout was used. A temperature response of $-1 \pm 0.12 \mathrm{~Hz} \mathrm{~K}^{-1}$ with respect to the first bending mode resonance frequency, $f_{\text {res }}$, was measured at room temperature, which is related to the temperature dependence of Young's modulus. ${ }^{6}$ An additional temperature controlled heat sink

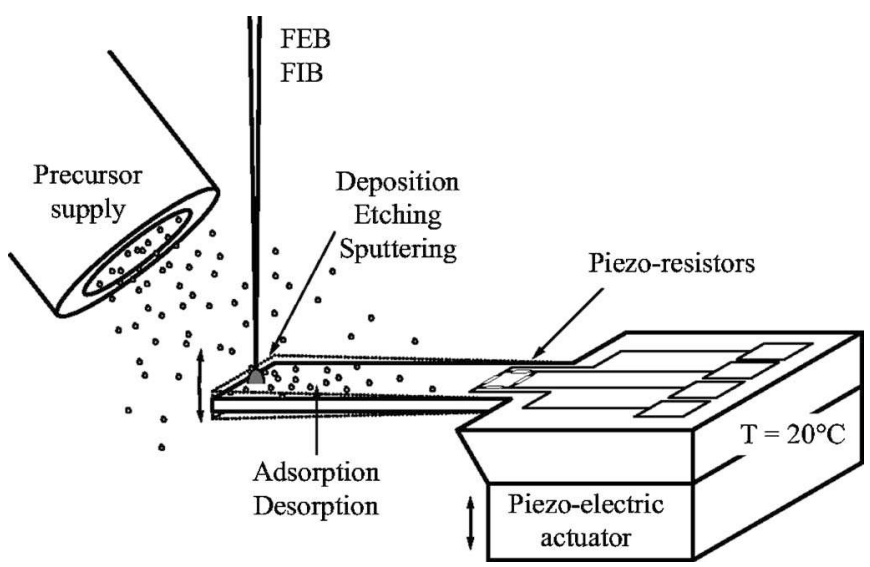

FIG. 1. Schematic diagram of the cantilever mass sensor for FIB/FEB induced process monitoring with local precursor supply from a microtube gas injection system. The mass added to or removed from the cantilever is detected as a negative or positive resonance frequency shift, respectively. 


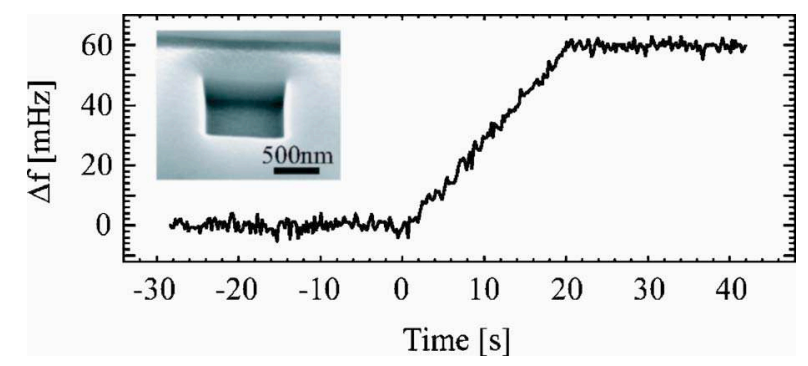

FIG. 2. Evolution of the cantilever resonance frequency during FIB (30 kV, $100 \mathrm{pA})$ sputtering of a $1 \times 1 \mu \mathrm{m}^{2}$ pit. Inset: SEM tilt view $\left(45^{\circ}\right)$ of the sputtered pit.

was integrated in order to stabilize the temperature of the sensor at $20^{\circ} \mathrm{C}$, compensating the heat due to dissipation of the applied bias power in the bridge.

FIB milling and FIB/FEB induced deposition experiments using the precursor trimethyl(methylcyclopentadienyl)platinum(IV) $\left(\mathrm{CH}_{3}\right)_{3} \mathrm{PtCpCH}_{3}$ (CAS: 94442-22-5) were carried out in a dual beam Nova 600 NanoLab instrument from FEI Company equipped with a liquid gallium ion and a Schottky field electron emitter. A gas injection system (GIS) supplied the precursor at an average molecule flux at the GIS exit of $J_{\text {exit }}=3.3 \times 10^{18}$ molecules $\mathrm{cm}^{-2} \mathrm{~s}^{-1}$ measured by mass loss of the precursor reservoir.

During typical deposition experiments (precursor on) a background chamber pressure of the order of $P=10^{-5} \mathrm{mbar}$ was measured and the fundamental mode quality factor of the cantilever, $Q$, was $>10000$. Phase locking of the excitation and response signals allowed continuous frequency and energy dissipation measurements with very high accuracy, ${ }^{17}$ i.e., the cantilever was continuously excited at its resonance frequency and the deflection amplitude was kept constant at values $<500 \mathrm{~nm}$. Deflection amplitudes in this order did negligibly affect the lateral and depth resolutions. Energy dissipation in the cantilever and to the environment is observed via the excitation amplitude. Running the phaselocked loop at a demodulation bandwidth of $\sim 1 \mathrm{~Hz}$ was an acceptable trade-off between signal-to-noise ratio and response time to follow the mass changes in FIB/FEB processing.

Mass response calibration was performed using the method proposed by Cleveland et al. ${ }^{7}$ The method is based on measuring the frequency shift induced by an added microsphere with known mass. This approach was considerably simplified by removing a defined volume of cantilever material using FIB milling. The resonance frequency shift of the cantilever sensor was continuously recorded during milling of a $1 \times 1 \mu \mathrm{m}^{2}$ pit by a focused $\mathrm{Ga}^{+}$ion beam $(30 \mathrm{kV}$, $50 \mathrm{pA}$ ) at the free end of the cantilever (see Fig. 2). Ga implantation during sputtering lead to added mass of $m_{\mathrm{Ga}}$ $=70 \mathrm{fg}$, estimated assuming a value of $6 \times 10^{16} \mathrm{~cm}^{-2}$ residual $\mathrm{Ga}$ at the bottom of the sputtered pit. $^{8}$ Using scanning electron microscopy (SEM) the volume, $V$, of the removed material was determined from the pit dimensions $\left(900 \times 900 \mathrm{~nm}^{2}\right.$ large, $340 \mathrm{~nm}$ deep $)$. The mass response at the cantilever end $(\Delta f / \Delta m)_{\text {end }}=\Delta f /\left(m_{\mathrm{Ga}}-V \cdot \rho_{\mathrm{Si}}\right)$ $=-103 \mathrm{mHz} \mathrm{pg}^{-1}$, where $\rho_{\mathrm{Si}}$ is the silicon density. The uncertainty of this in situ calibration is dominated by the visual determination of depth which allows a precision in the range of $\pm 5 \%$. Furthermore, a sputter yield of two atoms per ion was measured which compares well to reported values. ${ }^{9}$

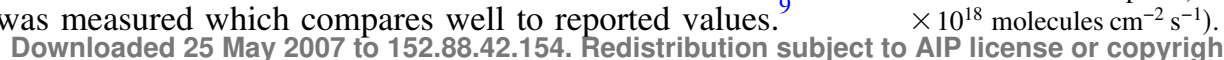

(a)

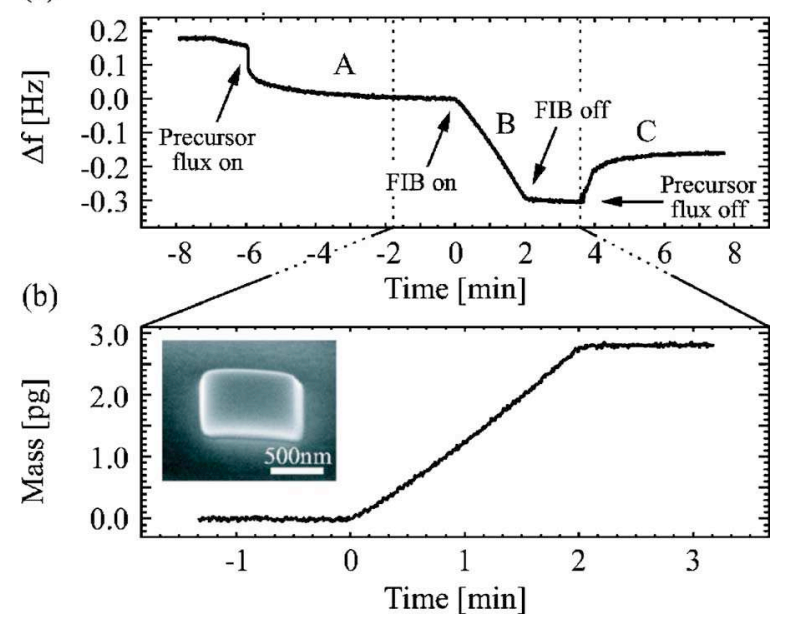

FIG. 3. (a) Precursor coverage controlled FIB induced deposition experiment from the precursor $\left(\mathrm{CH}_{3}\right)_{3} \mathrm{PtCpCH}_{3}$ : (A) Mass loading due to adsorption and (C) mass loss due to desorption. (B) FIB exposure of a 1 $\times 1 \mu \mathrm{m}^{2}$ rectangle. (b) Evolution of the FIB $(30 \mathrm{kV}, 10 \mathrm{pA})$ deposited mass corresponding to part (B) in (a). Inset: SEM tilt view $\left(52^{\circ}\right)$ of the FIB deposit.

The frequency rms noise level in the measurements of Fig. 2 is $1 \mathrm{mHz}$, which, in the limiting case of unity signalto-noise ratio, corresponds to a minimum detectable mass of $10 \mathrm{fg}$. Note that optimization of resonator dimensions offers mass resolution at the attogram ${ }^{10}$ and zeptogram scale. ${ }^{11}$

Figure 3 shows the response from the mass sensor during a complete FIB induced deposition experiment. Opening the $\left(\mathrm{CH}_{3}\right)_{3} \mathrm{PtCpCH}_{3}$ precursor reservoir lead to a negative frequency shift due to added mass from adsorption of precursor molecules on the cantilever surface. After several minutes, GIS and vacuum chamber achieved their equilibrium pressure as indicated by a constant and reproducible cantilever frequency shift of $\sim 150 \mathrm{mHz}$. The locally increased pressure at the GIS exit leads to higher damping of the cantilever, which is measured as an increase in dissipated excitation energy of $1 \%$. This has a negligible impact of $\sim 1 \mathrm{ppb}$ (parts per billion) on the frequency shift calculated by $\Delta f / f_{\text {res }}=1-\sqrt{1-1 /\left(2 Q^{2}\right)}$, for $Q=10000$.

The Monte Carlo simulated ${ }^{12}$ impinging precursor flux distribution, $J(x, y)$, is shown in Fig. 4. For low surface coverage, $\Theta$, nondissociative Langmuir adsorption predicts the

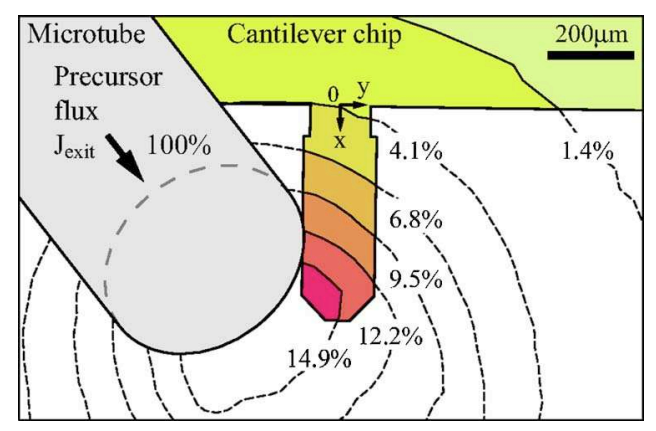

FIG. 4. (Color online) Top view of cantilever mass sensor and microtube (see Fig. 1). The microtube is $52^{\circ}$ inclined and its exit surface center is at a distance of $600 \mu \mathrm{m}$ relative to the cantilever chip plane. Monte Carlo simulated precursor isoflux lines show the impinging flux distribution on the cantilever chip (for clarity the contours are extended as dashed lines over the whole plane). Isoflux percentages are indicated $\left(J_{\text {exit }}=3.3\right.$ 
TABLE I. Comparison of FIB and FEB experiments with $\left(\mathrm{CH}_{3}\right)_{3} \mathrm{PtCpCH}_{3}$ (flux: $5 \times 10^{17}$ molecules $\mathrm{cm}^{-2} \mathrm{~s}^{-1}$ ): deposit density, $\rho_{\text {deposit }}$, Pt content, volume deposition yield, $Y_{V}$, mass deposition yield, $Y_{m}$, and deposited Pt atoms yields, $Y_{\mathrm{Pt}}$.

\begin{tabular}{lcc}
\hline \hline & FIB $(30 \mathrm{kV}, 5 \mathrm{pA})^{\mathrm{a}}$ & FEB $(5 \mathrm{kV}, 0.8 \mathrm{nA})^{\mathrm{b}}$ \\
\hline$\rho_{\text {deposit }}\left(\mathrm{g} \mathrm{cm}^{-3}\right)$ & 11.2 & 4.5 \\
$\mathrm{Pt}($ at. \%) & 42 & 15 \\
$Y_{V}\left(\mu \mathrm{m}^{3} \mathrm{nC}^{-1}\right)$ & 0.42 & $4.6 \times 10^{-3}$ \\
$Y_{m}\left(\mathrm{pg} \mathrm{nC}^{-1}\right)$ & 4.7 & $21 \times 10^{-3}$ \\
$Y_{\mathrm{Pt}}\left(\mathrm{Pt} /\right.$ ion, $\left.e^{-}\right)$ & 1.0 & $1.5 \times 10^{-3}$ \\
\hline
\end{tabular}

${ }^{\mathrm{a} D w e l l ~ t i m e: ~} 0.2 \mu \mathrm{s}$; loop time: $5.6 \mathrm{~ms}$.

${ }^{\mathrm{b}}$ Dwell time: $0.2 \mu \mathrm{s}$; loop time: $0.36 \mathrm{~ms}$.

mass distribution on the cantilever to be proportional to the flux distribution: $m(x, y)=m_{0} \Theta(x, y) \simeq m_{0} s J(x) \tau / n_{0}$, where $n_{0}$ is the density of a complete monolayer, $m_{0}$ the monolayer mass on the cantilever surface, $s$ the sticking probability, and $\tau$ the surface residence time. The frequency shift, $\Delta f$, is obtained by integrating the mass distribution and the positiondepending mass responsivity, $\Delta f / \Delta m$, along $x$,

$$
\Delta f=\frac{m_{0}}{L} \int_{0}^{L} \Theta(x) \frac{\Delta f}{\Delta m}(x) d x .
$$

For rectangular cantilevers with length, $L$ ( $\gg$ width), $\Delta f / \Delta m(x)=(x / L)^{3}(\Delta f / \Delta m)_{\text {end }}$. Averaging along $y$ results in insignificant responsivity deviations of $<2 \%$. ${ }^{13}$ From a $\left(\mathrm{CH}_{3}\right)_{3} \mathrm{PtCpCH}_{3}$ molecule size estimation from geometrical bond lengths the $100 \%$ monolayer density is found as $n_{0}$ $=2 \times 10^{14}$ molecules $\mathrm{cm}^{-2}$. Setting $s=1$, Eq. (1) was solved numerically and lead to a surface residence time of $\tau$ $\simeq 29 \mu \mathrm{s}$. This corresponds to the time scale of adsorption/ desorption equilibrium on the cantilever surface, which is instantaneous compared to the time scale of pressure variations in the chamber. In this calculation the chamber background flux to the cantilever was neglected which was $<5 \%$ of the average GIS-supplied flux to the top side. We calculated the precursor coverage in the present GIS configuration at the cantilever end: $\Theta \simeq s J(L) \tau / n_{0}=7 \%$ of a monolayer, taking $J(L)=0.149 J_{\text {exit }}$ from Fig. 4.

As material was added during FIB induced deposition $(30 \mathrm{kV}, 5 \mathrm{pA})$ in a $1 \times 1 \mu \mathrm{m}^{2}$ scan area the resonance frequency of the cantilever decreased. Figure 3(b) shows the mass converted FIB deposition sequence with a mass deposition rate of $23 \mathrm{fg} \mathrm{s}^{-1}$ in average. A small drift of $1.0 \mathrm{fg} \mathrm{s}^{-1}$ was corrected, which is due to the asymptotic approach of chamber pressure to equilibrium after opening the GIS. A deposited material density of $\rho_{\text {deposit }}=(11.2 \pm 1.1) \mathrm{g} \mathrm{cm}^{-3}$ was calculated from the total deposited mass of $2.8 \pm 0.14 \mathrm{pg}$ and the deposited volume of $0.25 \pm 0.01 \mu \mathrm{m}^{3}$ determined from the SEM images. Comparing the deposit density to $\rho_{\mathrm{Pt}}$ $=21.4 \mathrm{~g} \mathrm{~cm}^{-3}$ and typical $\mathrm{Ga}$ contamination obtained under similar conditions ${ }^{14}$ suggests that the material consists of 42 at. \% Pt, 29 at. \% Ga, and 29 at. \% carbonaceous matrix. The matrix density was assumed $\sim 1.5 \mathrm{~g} \mathrm{~cm}^{-3} .{ }^{15}$ An equivalent deposition experiment was performed using the FEB $(5 \mathrm{kV}, 0.8 \mathrm{nA})$ resulting in a deposit density of $\rho_{\text {deposit }}$ $=(4.5 \pm 0.5) \mathrm{g} \mathrm{cm}^{-3}$. A Pt content of 15 at. \% is estimated assuming the same matrix density as for the FIB deposit. Table I summarizes data extracted from the FIB and FEB experiments. These values were obtained for the specific set of experimental parameters as described in this letter. Variation of the ion/electron or the precursor flux was shown to have a strong influence on the FIB/FEB deposition rates and yields. ${ }^{16}$ In conclusion we have presented an experimental method for monitoring continuously in situ FIB/FEB induced deposition or removal processes with a resolution down to $10 \mathrm{fg}$. Precursor adsorption/desorption and mass deposition rate and yield have been measured using a cantilever-based mass sensor. As a result, precise measurements of material densities of deposits can be performed, which is essential for micro- and nanodevices to be used for mechanical applications.

The data shown in this letter was recorded using the Nanonis SPM Controller and Nanonis Phase-Locked Loop. The authors would like to acknowledge Nascatec GmbH for providing them with piezoresistive cantilevers.

${ }^{1}$ L. R. Harriott, A. Wagner, and F. Fritz, J. Vac. Sci. Technol. B 4, 181 (1986).

${ }^{2}$ T. Bret, I. Utke, P. Hoffmann, M. Abourida, and P. Doppelt, Microelectron. Eng. 83, 1482 (2006).

${ }^{3}$ G. C. Gazzadi and S. Frabboni, J. Vac. Sci. Technol. B 23, L1 (2005).

${ }^{4}$ A. Perentes, T. Bret, I. Utke, P. Hoffmann, and M. Vaupel, J. Vac. Sci. Technol. B 24, 587 (2006).

${ }^{5}$ A. D. Dubner and A. Wagner, J. Appl. Phys. 65, 3636 (1989).

${ }^{6}$ U. Gysin, S. Rast, P. Ruff, E. Meyer, D. W. Lee, P. Vettiger, and C. Gerber, Phys. Rev. B 69, 045403 (2004).

${ }^{7}$ J. P. Cleveland, S. Manne, D. Bocek, and P. K. Hansma, Rev. Sci. Instrum. 64, 403 (1993).

${ }^{8}$ J. G. Pellerin, G. M. Shedd, D. P. Griffis, and P. E. Russell, J. Vac. Sci. Technol. B 7, 1810 (1989).

${ }^{9}$ D. Santamore, K. Edinger, J. Orloff, and J. Melngailis, J. Vac. Sci. Technol. B 15, 2346 (1997).

${ }^{10}$ K. L. Ekinci, X. M. H. Huang, and M. L. Roukes, Appl. Phys. Lett. 84, 4469 (2004).

${ }^{11}$ Y. T. Yang, C. Callegari, X. L. Feng, K. L. Ekinci, and M. L. Roukes, Nano Lett. 6, 583 (2006).

${ }^{12}$ I. Utke, V. Friedli, S. Amorosi, J. Michler, and P. Hoffmann, Microelectron. Eng. 83, 1499 (2006).

${ }^{13}$ J. E. Sader, I. Larson, P. Mulvaney, and L. R. White, Rev. Sci. Instrum. 66, 3789 (1995).

${ }^{14}$ T. Tao, J. S. Ro, J. Melngailis, Z. L. Xue, and H. D. Kaesz, J. Vac. Sci. Technol. B 8, 1826 (1990).

${ }^{15}$ I. Utke, V. Friedli, J. Michler, T. Bret, X. Multone, and P. Hoffmann, Appl. Phys. Lett. 88, 031906 (2006).

${ }^{16}$ S. Lipp, L. Frey, C. Lehrer, B. Frank, E. Demm, S. Pauthner, and H. Ryssel, J. Vac. Sci. Technol. B 14, 3920 (1996).

${ }^{17}$ Nanonis GmbH, Switzerland, www.nanonis.com 